\title{
Macular phototoxicity after corneal cross-linking
}

This article was published in the following Dove Press journal:

Clinical Ophthalmology

\author{
Paulo Rodolfo Tagliari \\ Barbisan' \\ Marina Gonçalves Monteiro \\ Viturino' \\ Fernanda Maria Silveira \\ Souto' \\ Bo Tian ${ }^{2,3}$ \\ Roberto Damian Pacheco \\ Pinto' \\ Lucas Barasnevicius \\ Quagliato' \\ Maurício Abujamra \\ Nascimento' \\ Rosane Silvestre de Castro' \\ Carlos Eduardo Leite \\ Arieta' \\ 'Department of Ophthalmology, State \\ University of Campinas, Campinas, \\ São Paulo, Brazil; ${ }^{2}$ Angiogenesis \\ Laboratory, Department of \\ Ophthalmology, Massachusetts \\ Eye and Ear Infirmary, Harvard \\ Medical School, Boston, MA, USA; \\ ${ }^{3}$ Department of Ophthalmology \\ and Visual Sciences, University of \\ Massachusetts Medical School, \\ Worcester, MA, USA
}

Correspondence: Paulo Rodolfo Tagliari Barbisan

Department of Ophthalmology, State University of Campinas, Rua Floriano

Peixoto, 1853 - Alto da Boa Vista,

Ribeirão Preto - SP, I4025-220, Brazil

Tel +55 I69 9187 7107

Fax +55 I639II 1929

Email paulortbarbisan@gmail.com
Purpose: To assess potential vascular, structural, and functional changes to the macula in patients with keratoconus that underwent ultraviolet A (UVA)-riboflavin-mediated corneal collagen cross-linking (CXL) therapy.

Patients and methods: Seventeen eyes from 17 patients of age 16 years or older with keratoconus undergoing CXL treatment were studied. The same eye served as its own control (before CXL vs after CXL). Eyes were evaluated in terms of best-corrected visual acuity (BCVA), refractive error, intraocular pressure, Amsler grid, retinography, fluorescein angiography, autofluorescence, and spectral domain optical coherence tomography (SD-OCT) prior to CXL and 7 and 30 days after treatment. Multifocal electroretinography (mfERG) was recorded prior to and 7 days after CXL.

Results: Mean (SD) BCVA by logMAR chart was $0.47( \pm 0.12)$ pre-CXL, $0.55( \pm 0.15) 7$ days post-CXL $(P=0.57)$, and $0.46( \pm 0.10) 30$ days post-CXL $(P=0.87)$. Mean (SD) SD-OCT central macular thickness $(\mu \mathrm{m})$ was $253.62( \pm 20.9)$ pre-CXL, $260.5( \pm 18.7) 7$ days post-CXL $(P=0.48)$, and $256.44( \pm 21.6) 30$ days post-CXL $(P=0.69)$. In 12 eyes, mfERG revealed a statistically significant increase $(P=0.0353)$ in $\mathrm{P} 1$ latency $(\mathrm{ms})$ of ring four from the pre-CXL period $(39.45 \pm 2.05)$ to 7 days post-CXL $(41.04 \pm 1.28)$ period. Regression analysis showed that the increase in P1 latency was correlated with the increase in central macular thickness $(P=0.027)$. Furthermore, nine patients experienced a significant decrease in $P 1$ amplitudes of rings $1(P=0.0014), 2(P=0.0029)$, $3(P=0.0037), 4(P=0.0014)$, and $5(P=0.0012)$ from pre-CXL to 7 days post-CXL.

Conclusion: In this pilot study, most of the patients exhibited slight changes in their mfERG parameters and OCT thickness, despite a lack of vascular abnormalities observed on fluorescein angiography/autofluorescence imaging, no alteration in BCVA, and no reports of symptoms. These changes could, therefore, be categorized as a mild subclinical effect of the corneal crosslinking procedure.

Keywords: keratoconus, light damage, multifocal electroretinogram, UVA, phototoxicity, crosslinking

\section{Introduction}

Keratoconus is a bilateral and noninflammatory corneal degenerative disorder distinguished by paracentral corneal thinning and secondary ectasia. It results in irregular astigmatism with impaired vision. The disease occurs in all racial groups, equally affects males and females, and is often asymmetrical. Onset is typically at puberty and is progressive until the third to fourth decade of life. The etiology is not fully understood, and several different biochemical, physical, and genetic pathways have been implicated. The condition itself is a common final pathway for several different diseases. ${ }^{1,2}$ The reported prevalence and incidence vary, but a recent study calculated an annual incidence of 1:7,500 (13.3 cases per 100,000) and an estimated prevalence of $1: 375(265$ cases per 100,000$){ }^{3}$

Keratoconus management depends on the severity of the disease and the extent of irregular astigmatism. Eyeglasses, toric lenses, and rigid gas-permeable lenses are 
the treatment options for mild-to-moderate cases. ${ }^{2,4}$ Surgery (typically keratoplasty) becomes necessary when the disease progresses and in cases of contact lens intolerance, corneal scarring, and corneal thinning. ${ }^{2,5}$ However, none of these approaches treat the underlying causes of keratoconus or halt its progression.

Corneal collagen cross-linking (CXL) is a strengthening technology that alters the collagen matrix of the corneal stroma by creating riboflavin-ultraviolet A (UVA)-induced crosslinks. ${ }^{6,7}$ Riboflavin-catalyzed reactions generate free radicals that promote new collagen fibril bonds to a depth of $\sim 200-300 \mu \mathrm{m}$ and prevent further thinning of the corneal stroma by increasing the mechanical and biochemical rigidity and stability of stromal tissue. ${ }^{6-9} \mathrm{CXL}$ has been found to be effective in the stabilization of moderate corneal ectasia, in decreasing maximum keratometry, and in improving bestcorrected visual acuity (BCVA). 2,6,9,10 Numerous clinical studies have confirmed its efficacy in halting or aiding the regression of keratoconus. ${ }^{2,6,9,10}$

Phototoxic maculopathy is a retinal abnormality that generally affects patients after they engage in activities such as looking directly at a solar eclipse or welding without protection; it may also occur during accidents involving laser pointers or even after ocular surgery due to a photochemical reaction in light receptors. These patients may experience paracentral scotoma, metamorphopsia, or slight to moderate vision loss after exposure. ${ }^{11-13}$ The mechanisms by which light can cause damage to the retina are photothermal, photomechanical, and photochemical. ${ }^{14-16}$ Photothermal damage occurs through the transfer of radiant energy (a photon) from light to the retinal tissue, and common examples include transpupillary thermotherapy, laser photocoagulation, and micropulse diode laser. Photomechanical damage refers to tissue damage resulting from mechanical, compressive, or tensile forces generated by the rapid introduction of energy, such as that which is caused by the Nd:Yag laser. Photochemical damage to the retina is independent of mechanical and thermal retinal damage, and this type of damage occurs after UV light exposure or the clinical use of photodynamic therapy. ${ }^{17}$ UVA is potentially more hazardous to the retina than other wavelengths. ${ }^{18,19}$ Its use during the CXL procedure raises concerns over potential damage to ocular structures and to the retina in particular.

Combined UVA-riboflavin treatment is known to cause endothelial toxicity in thin corneas $(<400 \mu \mathrm{m})$ when the standard surface irradiation of $3 \mathrm{~mW} / \mathrm{cm}$ is used. ${ }^{2,6,20,21}$ Although in vitro studies have found that the shielding effect of riboflavin limits radiant transmission to inner ocular structures, few in vivo studies have been performed to assess morphological changes to the retina after UVA-riboflavin CXL, and there seem to be no studies in the literature that have analyzed functional damage to the retinal tissue. . $^{1822,23}$

The objective of this study is to assess potential vascular, structural, and functional changes to the macula in patients with keratoconus after UVA-riboflavin CXL therapy.

\section{Patients and methods}

This study evaluated 17 eyes from 17 patients who were 16 years of age or older, who had been diagnosed with keratoconus, and for whom treatment with corneal cross-linking had been indicated for at least one eye. All subjects were assessed in the Department of Ophthalmology of the Clinical Hospital of the State University of Campinas in Campinas, São Paulo State, Brazil. The same eye served as its own control (before CXL vs after CXL). Eyes were evaluated in terms of BCVA, refractive error, and intraocular pressure and using slit-lamp biomicroscopy, indirect ophthalmoscopy, the Amsler grid, color fundus imaging (retinography), fluorescein angiography (FA), autofluorescence (AF), and spectral domain optical coherence tomography (SD-OCT) prior to CXL and 7 and 30 days after the procedure. Multifocal electroretinography (mfERG) was recorded prior to and 7 days after CXL.

Patients were excluded if they were younger than 16 years of age, had any eye disease other than keratoconus, had a history of any ophthalmologic surgery in the eye being studied (including previous corneal cross-linking), were pregnant or breastfeeding, or exhibited any corneal opacity that would prevent clear vision of the retina or which would explain a loss of visual acuity. All participants provided informed written consent before participation; a parent or legal guardian signed the informed consent form when subjects were younger than 18 years of age. This study was approved by the Ethics Committee for Research on Human Subjects of the State University of Campinas and adhered to the Declaration of Helsinki.

\section{Corneal cross-linking procedure}

The UVA source was a solid-state device (X-Link Corneal Crosslinking System; Opto ${ }^{\circledR}$, São Carlos, Brazil) consisting of a $9 \mathrm{~mm}$ UVA array with a potentiometric voltage regulator. Wavelength was $370 \mathrm{~nm}$, and power was $3 \mathrm{~mW} / \mathrm{cm}^{2}$ or $5.4 \mathrm{~J} /$ $\mathrm{cm}^{2}$ when $1.5 \mathrm{~cm}$ from the cornea. After applying the blepharostat, a marker ( $9 \mathrm{~mm}$ in diameter) was used to remove the corneal epithelium in a central circle with a blunt spatula. A photosensitizing solution (0.1\% riboflavin - 400 MOSM; Ophthalmos $^{\circledR}$, São Paulo, Brazil) was applied every 3 minutes 
over a 30-minute period. Riboflavin was applied every 3 minutes during the 30 minutes of ultraviolet exposure. All of the surgeons who performed the procedure were at the same skill level. After treatment, patients were medicated four times per day with a topical antibiotic (moxifloxacin, Vigamox; Alcon ${ }^{\circledR}$, São Paulo, Brazil) and the eye was dressed with a soft therapeutic contact lens for 5 days.

\section{Optical coherence tomography}

To evaluate the anatomy of the macular region of the retina, SD-OCT imaging was performed using a spectral domain device (Spectralis HRA ${ }^{\circledR} \mathrm{OCT}$; Heidelberg Engineering, Heidelberg, Germany). The pupil was dilated using 1\% tropicamide and $10 \%$ phenylephrine drops. Patients were asked to gaze at the fixation light during the test, and foveal fixation was controlled by observing the retina through the infrared monitoring camera. Thickness of the macula region in the central ring was calculated using the retinal mapping software.

\section{Retinography, FA, and AF}

In order to perform retinal imaging and analyze the retinal vascular status of the macula and posterior pole of the retina, retinography and FA images were obtained using a fundus imaging device (Visucam ${ }^{\circledR}$ NM/FA; Carl Zeiss Meditec AG, Jena, Germany). The pupil was dilated using $1 \%$ tropicamide and $10 \%$ phenylephrine drops. AF images were obtained using the same device. Finally, a 10\% solution of sodium fluorescein dye was administered intravenously as a bolus at a dose of $7.7 \mathrm{mg} / \mathrm{kg}$ and serial retinal photographs were taken for 15 minutes.

\section{Multifocal electroretinography}

Roland RETI-port/scan 21 system ${ }^{\circledR}$ (Roland Consult, Brandenburg a.d. Havel, Germany) was used to measure mfERG in accordance with the International Society for Clinical Electrophysiology of Vision. The pupil was dilated using $1 \%$ tropicamide, and the cornea was anesthetized using $0.5 \%$ tetracaine. The fellow eye was occluded by a pad. Subjects were asked to fixate on the central cross. Patients with low visual acuity were asked to fixate steadily on the center of the screen. HK-Loop electrodes were used as active electrodes to record mfERG signals. The neutral and reference electrodes were mounted on the frontal-central canthus and the external canthus, respectively. The recording process took $\sim 10$ minutes, during which time cross-fixation lines were displayed on the screen. The recording procedure was repeated if there were artifacts from eye blinks or if ocular movements were recorded. The stimulus, which consisted of 103 hexagons covering a visual field of $30^{\circ}$, was presented on a high-resolution color monitor (PC-System 21" monitor, mean luminance $180 \mathrm{~cd} / \mathrm{m}^{2}$, high contrast). The eye-to-monitor distance was $33 \mathrm{~cm}$, which corresponded to a stimulated field of $\pm 30^{\circ}$ horizontally and $\pm 24^{\circ}$ vertically. The central $30^{\circ}$ of the retina was stimulated by flickering hexagons independently between black and white in a pseudorandomized binary sequence. Hexagon size was scaled with eccentricity to evoke focal responses of approximately the same amplitude in the response arrays. Each record was collected in five segments and lasted 45 seconds. Every sequence lasted $16.6 \mathrm{~ms}$ and was followed by a rest interval of $66.4 \mathrm{~ms}$ before the next active sequence was initiated. According to the eccentricities, the amplitudes and latencies were evaluated in five-ring retinal regions. The first-order mfERG response components were analyzed. The mean response density of the $\mathrm{P} 1$ amplitude (amplitude per unit of retinal area $\left[\mathrm{nV} / \mathrm{deg}^{2}\right]$ ) and the mean latency (ms) were determined. We selected the five most central hexagons to collect the central mfERG responses, which were averaged for the analysis. The mfERG examinations were performed before and 7 days after the CXL procedure.

\section{Statistical analysis}

Data were analyzed using GraphPad Prism, version 7.0a. A descriptive statistical analysis was performed, and distributions were summarized using proportions and means \pm SD. The D'Agostino-Pearson omnibus test was used to confirm the normality of the data. Patients' data on clinical features were analyzed using the paired $t$-test. The paired $t$-test was also used on P1 amplitude data before and after CXL. P1 latency was analyzed using the paired $t$-test and the pre- and post-CXL data. Correlation and regression analysis were also performed based on P1 latency. A significance level of $P<0.05$ was established for all of the analyses.

\section{Results}

Seventeen eyes from 17 subjects were included in the analysis. Mean (SD) age was $22.17( \pm 5)$ years (range 16-28 years). Sixty-three percent of the subjects were male. Mean (SD; Snellen) BCVA as per the logMAR chart was $0.47( \pm 0.12$; 20/59) pre-CXL, $0.55( \pm 0.15 ; 20 / 70) 7$ days post-CXL, and $0.46( \pm 0.10 ; 20 / 57) 30$ days post-CXL, with no significant differences between pre-procedure data and the data obtained 7 days after CXL $(P=0.57)$ or 30 days after CXL $(P=0.87)$. Mean (SD) near corrected visual acuity (Jaeger) was 2.42 $( \pm 0.60)$ pre-CXL, $3.4( \pm 0.63) 7$ days post-CXL, and 2.58 
Table I Demographic and clinical information on patients

\begin{tabular}{llllc}
\hline Parameters & Pre-CXL & 7 days post-CXL & P-value* & 30 days post-CXL \\
\hline Age (years) & $22.0 \pm 5.0$ & - & - & - \\
Gender (male, \%) & 63.64 & - & - & - \\
BCVA (LogMAR) & $0.47 \pm 0.12$ & $0.55 \pm 0.15$ & 0.57 & $0.46 \pm 0.10$ \\
Near visual acuity (Jaeger) & $2.42 \pm 0.60$ & $3.4 \pm 0.63$ & 0.19 & $2.58 \pm 0.61$ \\
SD-OCT thickness $(\mu \mathrm{m})$ & $253.62 \pm 20.9$ & $260.5 \pm 18.7$ & 0.48 & $256.44 \pm 21.6$ \\
\hline
\end{tabular}

Notes: Data presented as mean $\pm S D$. Independent $t$-test. $* P<0.05$ was considered significant.

Abbreviations: BCVA, best-corrected visual acuity; CXL, corneal collagen cross-linking; SD-OCT, spectral domain optical coherence tomography.

( \pm 0.61$) 30$ days post-CXL, with no significant differences between pre-procedure data and the data obtained 7 days after CXL $(P=0.19)$ or 30 days after CXL $(P=0.80)$. Mean (SD) SD-OCT central macular thickness $(\mu \mathrm{m})$ was $253.62( \pm 20.9)$ pre-CXL, $260.5( \pm 18.7) 7$ days post-CXL, and 256.44 ( \pm 21.6$)$ 30 days post-CXL, with no significant differences between pre-procedure data and the data obtained 7 days after CXL $(P=0.48)$ or 30 days after CXL ( $P=0.69$; Tables 1 and 2$)$. Subjects reported no changes on the Amsler grid test in the evaluations performed 7 and 30 days after CXL.

When the eyes were compared before and after CXL treatment, there were no changes in the fundus of the eye as determined using indirect ophthalmoscopy, biomicroscopy, or retinography, nor were there any abnormalities in the macular vascularity as determined using FA, or any changes to the retinal pigment epithelium based on AF imaging. Though the aforementioned changes in retinal thickness were observed, the intraretinal cysts, atrophy, outer retinal abnormalities, and other anatomical changes to the macular region typically seen in phototoxicity conditions ${ }^{16}$ were not observed via SD-OCT imaging when the eyes were compared before and after the CXL treatment. Intraocular pressure did not increase after the procedure or during the follow-up period.

In 12 eyes, mfERG revealed a statistically significant increase ( $P=0.0353)$ in P1 latency (ms) of ring 4 when preCXL values (39.45 \pm 2.05 ) were compared to values obtained 7 days post-CXL (41.04 \pm 1.28 ; Figure 1). Regression analysis in which P1 latency was a dependent variable of macular thickness showed that the increase in P1 latency in these subjects $(1.59 \pm 0.66)$ was correlated with the increase in central macular thickness in the SD-OCT scan $(P=0.027$; Figure 2; Table 3). Furthermore, nine patients were found to have a significant decrease in $\mathrm{P} 1$ amplitudes $\left(\mathrm{nV} / \mathrm{deg}^{2}\right)$ of rings $1-5$ when pre-CXL values were compared to the values 7 days

Table 2 Changes in BCVA (logMAR), CMT $(\mu \mathrm{m})$, and the five rings of latency as per the mfERG (ms) 7 days after CXL relative to pretreatment values

\begin{tabular}{llllllll}
\hline & LogMAR BCVA & CMT OCT $(\mu \mathrm{m})$ & Latency ring I & Latency ring 2 & Latency ring 3 & Latency ring 4 & Latency ring 5 \\
\hline 1 & -0.3 & +13 & -0.1 & +0.6 & +0.6 & +0.4 & -0.3 \\
2 & -0.5 & +15 & +2.8 & +1.9 & +2.1 & +3.6 & +1.8 \\
3 & -0.4 & +13 & +1.9 & 0 & +5.6 & +4.4 & +0.9 \\
4 & +0.1 & +5 & -0.3 & +0.6 & +0.2 & +1.9 & 0 \\
5 & 0 & +3 & -0.7 & -0.9 & -1.9 & 0 & 0 \\
6 & 0 & +5 & 0 & +0.3 & -0.1 & +1.8 & +0.3 \\
7 & +0.2 & +10 & +0.3 & -0.2 & 0 & 0 & 0 \\
8 & 0 & +2 & +1.6 & +0.8 & +0.7 & +1.9 & +0.9 \\
9 & -0.1 & +11 & +1.9 & -0.2 & +0.6 & +1.2 & +0.5 \\
10 & 0 & +2 & +1.9 & +0.5 & 0 & +0.9 & +0.8 \\
11 & -0.1 & +8 & -1.5 & 0 & +0.9 & +3.0 & 0 \\
12 & +0.2 & +6 & -1.9 & -0.9 & -0.3 & 0 & 0 \\
13 & 0 & +4 & -1.8 & -1.8 & -0.9 & +0.9 & -1.0 \\
14 & -0.3 & +12 & -0.7 & +0.9 & -1.0 & +1.0 & 0 \\
15 & -0.2 & +11 & -0.7 & -1.8 & -2.3 & +0.9 & -0.9 \\
16 & 0 & -2 & -0.6 & -1.9 & -0.9 & -2.8 & -1.9 \\
17 & 0 & -1 & -0.9 & -0.9 & -2.5 & 0 & 0 \\
Average & -0.08 & +6.88 & +0.14 & -0.17 & +0.04 & +1.30 & +0.06 \\
\hline
\end{tabular}

Notes: Data presented as changes in total values in each eye. Bold values represent the average result of each column.

Abbreviations: BCVA, best-corrected visual acuity; CMT, central macular thickness; CXL, corneal collagen cross-linking; mfERG, multifocal electroretinography; OCT, spectral domain optical coherence tomography. 


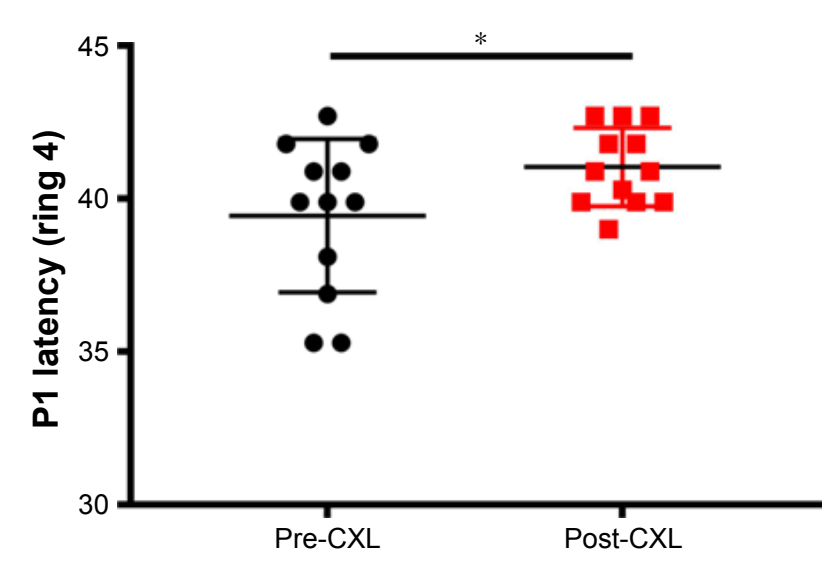

Figure I PI latency (ms) of ring 4 region. Data from before CXL and 7 days after CXL. Paired $t$-test.

Notes: $* P<0.05$. Data presented as mean $\pm S D$.

Abbreviation: $\mathrm{CXL}$, corneal collagen cross-linking.

after CXL (Figure 3; Table 4). Mean amplitudes in each ring (pre-CXL $\pm \mathrm{SD}$, post-CXL $\pm \mathrm{SD}, P$-value) were as follows: ring

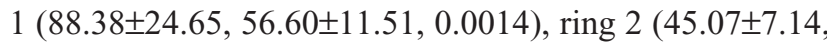
$35.72 \pm 7.20,0.0029)$, ring $3(29.39 \pm 3.65,25.15 \pm 3.50$, $0.0037)$, ring $4(19.55 \pm 4.16,15.99 \pm 3.09,0.0014)$, and ring $5(13.48 \pm 2.10,11.37 \pm 2.19,0.0012)$.

\section{Discussion}

Corneal collagen CXL is the only treatment that affects the progression of keratoconus and has been found to be effective in the stabilization of moderate corneal ectasia, in decreasing maximum keratometry, and in improving BCVA. ${ }^{2,6,9,10}$ In an attempt to strengthen the corneal stroma, the eye is exposed to a direct source of UVA light, a process which creates riboflavin-UVA-induced crosslinks on the cornea. It is unknown whether the UVA light to which the patient is exposed during the procedure can pass through ocular structures and cause phototoxicity damage to the retina.

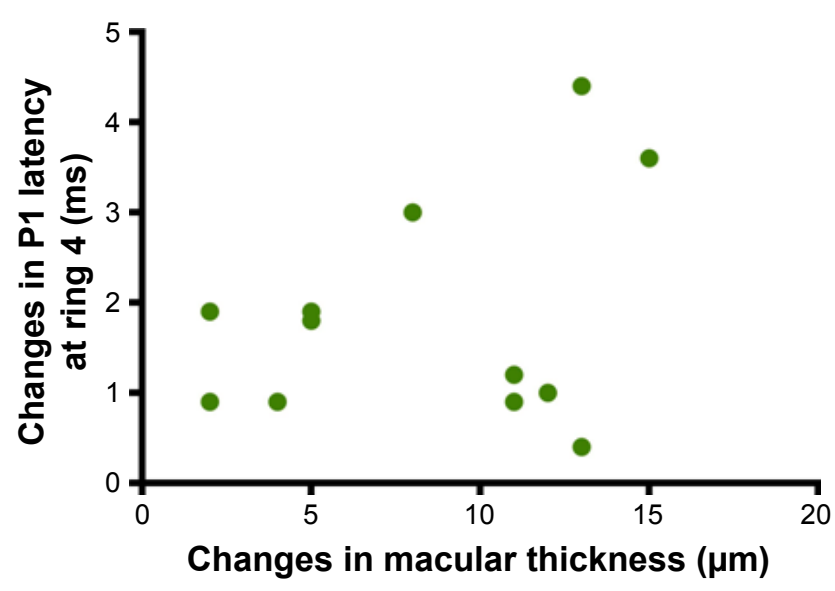

Figure 2 Scatter plot for changes in macular thickness and PI latency.
Table 3 Regression analysis of PI latency (ms) as a dependent variable with increase in macular thickness as determined by mfERG

\begin{tabular}{lll}
\hline & Mean \pm SD & $P$-value \\
\hline PI latency increase & $1.59 \pm 0.66$ & $0.027^{\mathrm{a}}$ \\
\hline
\end{tabular}

Note: aLinear regression.

Abbreviation: mfERG, multifocal electroretinography.

It is known, however, that UVA and other light wavelengths can produce phototoxic maculopathy, ${ }^{24}$ a fact which raises concern of retinal damage from exposure to UVA light during the CXL procedure. Though in vitro studies have shown that the shielding effect of riboflavin limits radiant transmission on inner ocular structures, few in vivo studies have been performed to assess the morphological changes to the retina after UVA-riboflavin CXL, and no studies were found in the literature on functional damage to the retinal tissue. ${ }^{13,22,23}$

In this study, 17 eyes were evaluated to determine any changes to BCVA, structural changes to the retina (SD-OCT, $\mathrm{AF}$ ), vascular status (FA), and function (electrophysiology with mfERG) before and after exposure to UVA during the CXL procedure. All patients received slit-lamp evaluations before the data were collected to ensure that there were no opacities on the ocular structures (corneal edema, scars, or abnormal surface integrity) that could represent the underlying cause of any abnormalities.

The results showed a slight decrease in BCVA and near vision acuity 7 days after the corneal CXL procedure; acuity, however, was found to have been recovered by day 30 . SD-OCT imaging of the macula region showed increased thickness 7 days after CXL and similarly returned to original

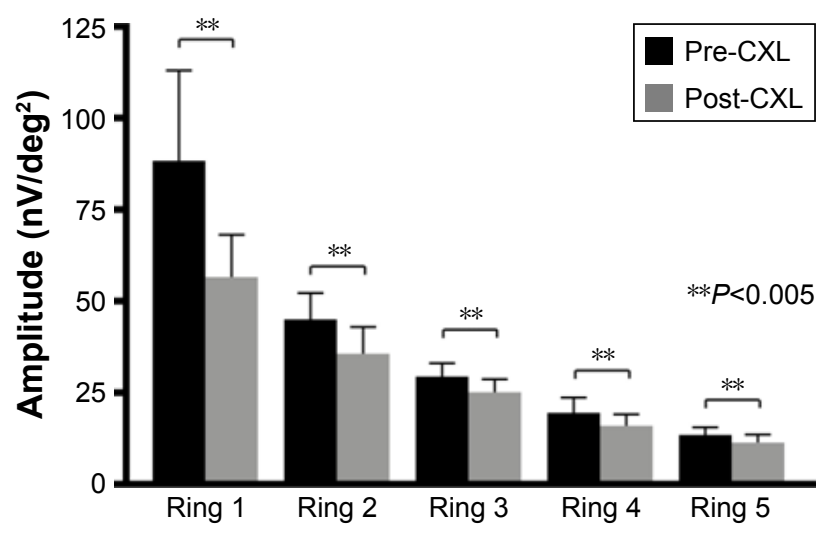

Figure $3 \mathrm{PI}$ amplitude $\left(\mathrm{nV} / \mathrm{deg}^{2}\right)$ of rings I-5.

Notes: Data from before crosslinking (pre-CXL) and from 7 days after crosslinking (post-CXL). Paired $t$-test. Data presented as mean \pm SD.

Abbreviation: CXL, corneal collagen cross-linking. 
Table 4 Changes in amplitudes of rings $1-5$ as per the mfERG $\left(\mathrm{nV} / \mathrm{deg}^{2}\right) 7$ days after $\mathrm{CXL}$ relative to pretreatment values

\begin{tabular}{llllll}
\hline Patients & Ring I & Ring 2 & Ring 3 & Ring 4 & Ring 5 \\
\hline $\mathrm{I}$ & -17.63 & -0.97 & -8.74 & -5.07 & -1.59 \\
2 & -39.14 & -3.0 & $-2.6 \mathrm{I}$ & -4.35 & -1.8 \\
3 & -5.1 & -11.33 & -2.88 & -3.5 & +1.99 \\
4 & -60.76 & $-11.3 \mathrm{I}$ & -4.89 & -7.8 & -2.14 \\
5 & -49.15 & -16.62 & -0.47 & -0.6 & -4.76 \\
6 & -45.45 & -5.86 & -4.73 & -3.19 & -1.15 \\
7 & -42.75 & -2.99 & +4.3 & -4.82 & -1.36 \\
8 & +32.71 & -4.06 & +4.55 & -4.02 & +1.98 \\
9 & -16.01 & -13.32 & +0.06 & -1.29 & -0.59 \\
10 & +29.5 & +3.3 & +1.69 & +3.32 & +1.21 \\
11 & +15.49 & +7.43 & -5.08 & +0.63 & -0.30 \\
12 & +7.04 & +5.42 & -8.72 & +5.47 & -3.63 \\
13 & -11.61 & -1.06 & -2.43 & -3.02 & -2.0 \\
14 & +28.23 & +2.11 & -2.66 & -1.68 & +0.22 \\
15 & +3.46 & +11.61 & +7.06 & +4.34 & +3.89 \\
16 & +24.08 & +6.44 & +5.64 & +4.06 & +2.72 \\
17 & +29.27 & +8.32 & -10.03 & -7.22 & +0.61 \\
Average & -7.34 & -1.40 & -1.76 & -1.69 & -0.39 \\
\hline
\end{tabular}

Note: Data presented as changes in total values in each eye.

Abbreviations: CXL, corneal collagen cross-linking; mfERG, multifocal electroretinography.

levels by day 30 . None of the differences between the groups was statistically significant.

There were no changes in the fundus of the eye as assessed using indirect ophthalmoscopy, biomicroscopy, or retinography. No changes or abnormalities in macular vascularity were observed in the FA, nor was any retinal pigment epithelium damage detected by AF imaging. Moreover, typical signs of phototoxicity, such as disruption of the inner segment/outer segment junction layer, intraretinal cists, or atrophy of the outer nuclear layer, were not detected by SD-OCT imaging.

All patients underwent mfERG, so that changes in electrical activity and function of the macular region could be assessed. Changes are usually defined as an increase in P1 latency and a decrease in P1 amplitude. According to the literature, symptoms of light damage to the retina, such as peri-central scotoma, metamorphopsia, or slight to moderate vision loss after light exposure, are typically perceived by patients within hours or days. ${ }^{11}$ For this reason, electrophysiological function of the macula was evaluated before and 7 days after CXL. Most patients were found to have experienced a significant increase in P1 latency (ms) of ring 4 (Figure 1). This change was correlated with an increase in central macular thickness (Figure 2; Table 3). There is no well-known correlation between visual acuity and the loci of mfERG rings; however, based on the location of ring 4 (macular periphery), neither a change in BCVA nor the perception of symptoms would be expected in these cases. Furthermore, because BCVA is usually low among keratoconus patients for whom CXL is indicated, any mild changes in vision resulting from light damage are unlikely to be detected. Therefore, changes to the anatomy or function of the eye could represent phototoxicity damage. In this study, nine subjects $(52.9 \%)$ also exhibited a significant decrease in P1 amplitudes of rings 1-5 (Figure 3), but these changes were not correlated with visual acuity or retinal thickness.

The limitations of the study are the number of patients (due to their difficulties in attending many follow-up appointments) and the lack of an electrophysiology evaluation 30 days after the procedure to analyze further disturbances or recoveries from the changes noticed on day 7 .

According to other studies, the retina is protected by the absorption capacity of the cornea and crystalline lens during CXL treatment. ${ }^{18}$ Although the procedure involved long-term (30 minutes of) exposure to UVA light, this study showed that the changes observed using SD-OCT and mfERG are not similar in nature or magnitude to other phototoxicity conditions described previously. This difference could be explained by the protective features of the anterior segment ${ }^{25,26}$ and the low UVA light intensity of the procedure relative to the intensity associated with typical phototoxicity conditions. The SD-OCT findings also reflected an increase in central macular thickness, which is the opposite of the typical thinning seen in outer retinal abnormalities. The hypothesis here is that this increase in macular thickness could be explained by a mild and self-limiting inflammatory reaction to the procedure and to UV light exposure. This reaction was evident 7 days after the procedure, but the patients were found to have recovered in the evaluations performed 30 days after CXL. Further studies are needed to prove or disprove this hypothesis. Even so, despite the lack of major differences between the groups in terms of BCVA, near vision acuity, and SD-OCT thickness, the slight changes seen during mfERG could raise concerns over subclinical macular disturbance after UVA light exposure during CXL. Given the results of a previous study, which showed the standard mfERG is largely shaped by on- and off-bipolar cell activity with more limited contributions from the photoreceptors and inner retinal (eg, amacrine and ganglion) cells, ${ }^{27}$ the hypothesis resulting from the current study is that this atypical presentation of phototoxicity would not produce consistent changes to BCVA, major visual symptoms, or structural changes that would be evident in SD-OCT imaging.

In addition, this is the first study found in the literature in which the anatomy, vascularity, and electrophysiology of 
the macula have been analyzed after exposure to UVA light from the corneal cross-linking procedure. Larger studies with long-term data are needed to further address possible phototoxicity of the retina and the safety of corneal crosslinking for keratoconus patients.

\section{Conclusion}

Continuous UVA light exposure during CXL therapy raises concerns over potential phototoxicity damage to the retina. This study is the first to analyze potential vascular, structural, and functional changes to the macula in patients with keratoconus who have been exposed to UVA light during cross-linking therapy. Most of the patients studied herein experienced slight changes in central macular thickness (as per SD-OCT) and in mfERG parameters, despite the absence of typical phototoxicity-related alterations seen in other conditions resulting from UV light exposure damage, lack of vascular abnormalities as determined by FA/AF imaging, and lack of significant changes in BCVA or near visual acuity and no report of symptoms. The changes seen could, therefore, be categorized as an early and mild subclinical effect on macular function after the corneal cross-linking procedure.

\section{Acknowledgment}

This project was supported by the São Paulo Research Foundation (FAPESP) under grant number 2015/23237-1.

\section{Disclosure}

The authors report no conflicts of interest in this work.

\section{References}

1. Rabinowitz YS. Keratoconus. Surv Ophthalmol. 1998;42(4):297-319.

2. Mas Tur V, Macgregor C, Jayaswal R, O'Brart D, Maycock N. A review of keratoconus: diagnosis, pathophysiology, and genetics. Surv Ophthalmol. 2017;62(6):770-783.

3. Godefrooij DA, de Wit GA, Uiterwaal CS, Imhof SM, Wisse RP. Agespecific incidence and prevalence of keratoconus: a nationwide registration study. Am J Ophthalmol. 2017;175:169-172.

4. Mandell RB. Keratoconus. In: Mandell RB, editor. Contact Lens Practice. 4th ed. Springfield: Charles C Thomas; 1988:824-849.

5. Kirkness CM, Ficker LA, Steele AD, Rice NS. The success of penetrating keratoplasty for keratoconus. Eye. 1990;4(Pt 5):673-688.

6. Suri K, Hammersmith KM, Nagra PK. Corneal collagen cross-linking: ectasia and beyond. Curr Opin Ophthalmol. 2012;23(4):280-287.

Clinical Ophthalmology

\section{Publish your work in this journal}

Clinical Ophthalmology is an international, peer-reviewed journal covering all subspecialties within ophthalmology. Key topics include: Optometry; Visual science; Pharmacology and drug therapy in eye diseases; Basic Sciences; Primary and Secondary eye care; Patient Safety and Quality of Care Improvements. This journal is indexed on
7. Mccall AS, Kraft S, Edelhauser HF, et al. Mechanisms of corneal tissue cross-linking in response to treatment with topical riboflavin and long-wavelength ultraviolet radiation (UVA). Invest Opthalmol Vis Sci. 2010;51(1):129-138.

8. Wollensak G, Spoerl E, Seiler T. Stress-strain measurements of human and porcine corneas after riboflavin-ultraviolet-A-induced crosslinking. J Cataract Refract Surg. 2003;29(9):1780-1785.

9. Raiskup-Wolf F, Hoyer A, Spoerl E, Pillunat LE. Collagen crosslinking with riboflavin and ultraviolet-A light in keratoconus: long-term results. $J$ Cataract Refract Surg. 2008;34(5):796-801.

10. Hersh PS, Greenstein SA, Fry KL. Corneal collagen crosslinking for keratoconus and corneal ectasia: one-year results. $J$ Cataract Refract Surg. 2011;37(1):149-160.

11. Rodríguez-Marco NA, Andonegui-Navarro J, Compains-Silva E, et al. Optical coherence tomography and macular phototoxicity. Arch Soc Esp Oftalmol. 2008;83(4):267-272.

12. Garg SJ, Martidis A, Nelson ML, Sivalingam A. Optical coherence tomography of chronic solar retinopathy. Am J Ophthalmol. 2004; 137(2):351-354.

13. Bechmann M, Ehrt O, Thiel MJ, Kristin N, Ulbig MW, Kampik A. Optical coherence tomography findings in early solar retinopathy. $\mathrm{Br}$ J Ophthalmol. 2000;84(5):547-548.

14. Glickman RD. Phototoxicity to the retina: mechanisms of damage. Int J Toxicol. 2002;21(6):473-490.

15. Solley WA, Sternberg P. Retinal phototoxicity. Int Ophthalmol Clin. 1999;39(2):1-12.

16. Verma L, Venkatesh P, Tewari HK. Phototoxic retinopathy. Ophthalmol Clin North Am. 2001;14(4):601-609.

17. Youssef PN, Sheibani N, Albert DM. Retinal light toxicity. Eye. 2011; 25(1):1-14.

18. Goldich Y, Marcovich AL, Barkana Y, Avni I, Zadok D. Safety of corneal collagen cross-linking with UV-A and riboflavin in progressive keratoconus. Cornea. 2010;29(4):409-411.

19. Ham WT, Mueller HA, Sliney DH. Retinal sensitivity to damage from short wavelength light. Nature. 1976;260(5547):153-155.

20. Wollensak G, Spoerl E, Wilsch M, Seiler T. Endothelial cell damage after riboflavin-ultraviolet-A treatment in the rabbit. J Cataract Refract Surg. 2003;29(9):1786-1790.

21. Kymionis GD, Portaliou DM, Diakonis VF, et al. Corneal collagen cross-linking with riboflavin and ultraviolet-A irradiation in patients with thin corneas. Am J Ophthalmol. 2012;153(1):24-28.

22. Spoerl E, Mrochen M, Sliney D, Trokel S, Seiler T. Safety of UVAriboflavin cross-linking of the cornea. Cornea. 2007;26(4):385-389.

23. Grewal DS, Brar GS, Jain R, et al. Corneal collagen crosslinking using riboflavin and ultraviolet-A light for keratoconus: one-year analysis using Scheimpflug imaging. J Cataract Refract Surg. 2009;35(3): 425-432.

24. Glickman RD. Ultraviolet phototoxicity to the retina. Eye Contact Lens. 2011;37(4):196-205.

25. Söderberg PG, Talebizadeh N, Yu Z, Galichanin K. Does infrared or ultraviolet light damage the lens? Eye. 2016;30(2):241-246.

26. Löfgren S, Söderberg PG. Lens lactate dehydrogenase inactivation after UV-B irradiation: an in vivo measure of UVR-B penetration. Invest Ophthalmol Vis Sci. 2001;42(8):1833-1836.

27. Hood DC, Frishman LJ, Saszik S, Viswanathan S. Retinal origins of the primate multifocal ERG: implications for the human response. Invest Ophthalmol Vis Sci. 2002;43(5):1673-1685.

\section{Dovepress}

PubMed Central and CAS, and is the official journal of The Society of Clinical Ophthalmology (SCO). The manuscript management system is completely online and includes a very quick and fair peer-review system, which is all easy to use. Visit http://www.dovepress.com/ testimonials.php to read real quotes from published authors. 